$$
\begin{aligned}
& \alpha{\overline{\langle\mathbf{J}\rangle_{e+}}}=-0.36 \pm 0.20 \\
& \alpha{\overline{\langle\mathbf{J}\rangle_{e}}}_{e^{-}}=-0.13 \pm 0.26
\end{aligned}
$$

The two decay modes of $K+$-meson $(\theta$-mode, $\pi++$ $\pi^{0}$, and $\tau$-mode, $\pi^{+}+\pi^{+}+\pi^{0}$ ) provide additional evidence for violation of parity. The final state consisting of $\pi^{+}+\pi^{0}$ mesons has spin-parity assignments $0+, 1-, 2+$, etc. It is easy to show that corresponding assignments for the $\left(\pi^{+}+\pi^{+}+\pi^{-}\right)$ state are $0-, 1+, 1-, 2+$, etc. There is considerable evidence that the spin of the $K$-meson is zero. Thus, $K$-particles decay through two modes, with opposite intrinsic space-reflexion properties.

For the theoretical interpretation of 'strange'particle decays there is nothing analogous to the neutrino to carry the blame for violation of parity. It may be recalled that it was the possibility of making the gauge transformation $\psi_{\nu} \rightarrow \gamma_{s} \psi_{v}$ for the neutrino (on account of its zero-mass) which led to the 2-state theory. One would like to have something analogous to this transformation for 'strange'particles.

Already in neutrino physics there are indications that the transformation, $\psi_{\nu} \rightarrow \gamma_{5} \psi_{v}$, is not enough. There is the obstinate fact in $\mu$-decay that $g_{A}=$ $-g_{V}$. One can perhaps find a natural explanation for this by generelizing the neutrino gauge to electrons or $\mu$-mesons, in the following manner. We postulate that all $\mu$-interactions or electron-interactions are invariant for the transformations

$$
\begin{gathered}
\psi_{e} \rightarrow \gamma_{s} \psi_{e}, \quad m_{e} \rightarrow-m_{e} \\
\text { or } \psi_{\mu} \rightarrow \gamma_{s} \psi_{\mu}, \quad m_{\mu} \rightarrow-m_{\mu}
\end{gathered}
$$

In so far as the electro-magnetic interaction is not changed by these (mass-reversal) transformations, invariance for mass-reversal appears as a universal law for all light particles.

Unfortunately, the current theories of strong interactions are not mass-reversal invariant. J. Sakurai ${ }^{1 \theta}$ has, however, proposed that as a start towards obtaining the interaction for 'strange'- particle decays one may postulate that all 'strange'particle decays arise from weak couplings of the type $\overline{(\Lambda} N) \overline{(N} N)$, where $N$ stands for nucleons, and that mass-reversal applies to these. One then obtains the result that there exists a universal 4-field inter. action between Fermi fields with equal mixtures of $A$ and $V$. In the particular case of $\Lambda$-decay, this gives $\alpha=0.89$. This unified point of view appears attractive although it suffers from a number of theoretical objections. The chief experimental evidence against it, however, is the result of $e-v$ correlation experiments on helium-6 decay, mentioned before.

This discussion would not be complete without some mention of the so-called $P C T$ theorem ${ }^{11}$. It can be shown that invariance of a theory for Lorentz rotations automatically implies its invariance under each of the six products $P C T$, $T P C, P T C$, etc., where $P$ stands for parity, $C$ for charge-conjugation and $T$ for time-reflexion invariance. It is clear that -besides parity-at least one other invariance must be violated in weak decays. It can be shown that all experiments imply $C$-violation. Weak interactions are not charge-conjugation symmetric. It is still an open question whether or not time-reflexion symmetry holds in these processes.

A. SALAM

1 Wu, C. S., Ambler, E., Hayward, R. W., Hopper, D. D. , and Hudson, K. P., Phys. Rev., 105, 1413 (1957). Garwin, R. L., Lederman, L. M., and Weinrich, M., Phys. Rev., 105,
1415 (1957). Friedman, J. I., and Telegdi, V. L., ibid., 105, 1681
(1957). ${ }^{3}$ Lee, T. D., and Yang, C. N., Phys. Rev., 104, 259 (1956).

"See, for example, Boehm, F., Novey, T. B., Barnes, C. A., and Stech, B., Phys. Rev. (in the press).

Culligan, G., Frank, S. G. F., Holt, J. R., Kluyver, J. C., and Massam, T., Nature, 180, 751 (1957).

- Salam, A., Nuovo Cirn., 5, 299 (1957). Landau, L. D., Nuc. Phys., 3, 127 (1957). Lee, T. D., and Yang, C. N., Phys. Rev., 3, 127 (1957).

${ }^{7}$ Crowe, K., Bull. Arn. Phys. Soc., 2, 206 (1957).

- Rustad, B. M., and Ruby, S. L., Phys. Rev., 103, 991 (1955).

- Stelnberger, J., et al., Phys. Rev. (in the press).

to Sakurai, J. J., Phys. Rev. (in the press). Pauli, W. in "Niels Bohr and Development of Physics" (Pergamon
Press, London, 1955).

\title{
VACANCIES AND OTHER POINT DEFECTS IN METALS AND ALLOYS
}

$I^{\mathrm{N}}$ N recent years there has been considerable interest in the behaviour of lattice vacancies, interstitial atoms and other more complex point defects in metals. In order to survey this subject, the Metal Physics Committee of the Institute of Metals invited six papers which would form the basis of a symposium. By kind permission of Sir John Cockcroft, the symposium was held at the Atomic Energy Research Establishment, Harwell. On December 9, following visits to the reactors DIDO and PLUTO and some laboratories, an introductory lecture was given by Prof. R. W. K. Honeycombe (University of Sheffield). He showed that a knowledge of the characteristics of point defects is necessary for the understanding of a wide range of metallurgical phenomena, including quench- and irradiationhardening, fatigue and creep.

On December 10 the six invited papers were briefly presented to an audience of about three hundred, and general discussion followed. Dr. A. H. Cottrell
(Atomic Energy Research Establishment, Harwell) considered how point defects can cause or contribute to hardening and softening processes in metals at temperatures lower than those at which conventional high-temperature creep occurs. The most spectacular hardening is that produced by quenching very pure metals from temperatures near the melting point. If the quench is sufficiently rapid, the high-temperature equilibrium concentration of vacancies remains randomly dispersed, and there is little or no hardening. Then if the metal is warmed sufficiently for the vacancies to agglomerate or migrate to dislocations, considerable hardening can occur. The detailed interpretation of quenching experiments was repeatedly shown to be of crucial importance for the understanding of the behaviour of lattice vacancies. When a metal is bombarded by energetic particles, occasional collisions lead to the knocking of atoms from lattice sites and the consequent production of equal numbers of vacancies and interstitials. The 
hardening produced by irradiation is often considerable, but the processes are apparently complex. It appears that both the initiation of plastic deformation and its continuation are usually made more difficult, indicating that point defects do interact with dislocations to give 'source hardening', and with each other to give general lattice hardening. During plastic deformation of annealed metals, lattice vacancies are produced and in certain circumstances can lead to readily observed increases in local diffusion rates. This is particularly prominent in fatigue, and it is probable that there is also a characteristic fatigue-hardening produced by point defects which more resembles quench- and irradiation-harder,ing than work-hardening.

The effects of point defects on some physical properties afford means for the study of their generation, movement and disappearance. The existing theoretical and experimental evidence was summarized by Dr. T. Broom and R. K. Ham (University of Birmingham). It was concluded that while the measurement of electrical resistivity is often experimentally simple, the uncertaintios in interpretation are often such as to render more desirable the rather difficult measurement of stored-energy. The other properties considered were density, thermo-electric power, magnetic behaviour, thermal conductivity and nuclear magnetic resonance. The most useful experiments are obviously those in which the changes of many properties are studied simultaneously.

Dr. W. M. Lomer (Atomic Energy Research Establishment, Harwell) considered the transport of atoms through metallic lattices in various types of diffusion processes, of which vacancy diffusion is the most important. In many face-centred-cubic metals the details are well understood, but in other lattices the 'structure' of a vacancy and of other point defects remains uncertain and hinders agreement between theory and experiment. Vacancies in alloys usually associate preferentially with one species of atom, and useful information can be gained about this indirectly from quenching experiments and directly from tracer diffusion measurements in homogeneous alloys. It is probable that non-equilibrium concentrations of vacancies introduced into homogeneous alloys, for example, by irradiation, can only lead to accelerated diffusion over very small distances because of the density of vacancy traps. Chemical diffusion in inhomogeneous systems is more complex on account of the complication of the gradient of chemical potential. Briefly, each species of atom will migrate at a different rate along the gradient leading to shifting of diffusion interfaces, to 'vacancy pumping' and frequently to formation of porosity due to local vacancy supersaturations. The detailed behaviour of sinks (and sources) for vacancies is important not only in such systems but also in vacancy-creep and in sintering.

The activation energy for creep at high temperatures has frequently been found to be very close to that required for self-diffusion. In a general account of high-temperature mechanical properties, Dr. D. McLean (National Physical Laboratory) showed how this is accounted for by mechanisms involving climb of dislocations caused by their absorption of thermal vacancies. The relative rates of creep in various metals can be qualitatively explained by considering the structure of the dislocations involved and whether they can readily act as sinks for vacancies. Alloying usually reduces the rate of secondary creep, and it is possible by simple calculations to indicate what modifications to the dislocation climb model may occur in particular cases. During the later stages of creep tests, voids frequently appear at grain-boundaries. The hypothesis that these are formed by the condensation of vacancies is confirmed after critical examination of the conditions for nucleation and growth.

Dr. P. L. Pratt (University of Birmingham) contributed a paper discussing the role of point defects in determining the mechanical properties of ionic crystals. Historically, there is good precedent for such studies casting light on similar properties in metals, and it was shown that quench-hardening, generation of vacancies by moving dislocations, dislocationpinning and vacancy cluster hardening all occur in crystals of sodium chloride. However, an important contribution to hardening in ionic crystals arises from the positive charge carried by dislocations which itself is a consequence of differing energies of formation of positive and negative ion vacancies. The line charges are screened by clouds of oppositely charged vacancies and this causes additional dislocation-pinning.

The last paper of the symposium, by E. C. Williams and P. C. S. Hayfield (Imperial Chemical Industries, Metals Division, Birmingham), dealt with the character of the metallic surface conceived of as containing an abnormal concentration of point defects and the influence that this has upon dry corrosion. Many new experimental results were presented from work in progress, and it was considered that they could best be explained by introducing into the theory of oxidation the concept of the trapping of electrons by localized structural defects.

In opening the general discussion, Prof. R. Maddin (University of Pennsylvania) gave new results for the hardening of polycrystalline copper by quenching. Specimens which had been rapidly quenched to room temperature could be hardened by slight warming, but heating at a higher temperature caused softening. Both processes occurred with an activation energy of $0.7 \mathrm{eV}$. and were believed to be associated with the movement of single vacancies. In a new theoretical interpretation of the effects of quenching on the electrical resistivity of gold it was proposed that quenching from low temperatures $\left(\sim 500^{\circ}\right.$ C. $)$ produced single vacancies but that during quenching from high temperatures $\left(\sim 900^{\circ}\right.$ C.) some pairs, clusters and collapsed clusters (sessile dislocations) were created and then later modified appreciably the kinetics of annealing-out of the extra resistivity. Other experimental results were presented on the effect of straining and rate of strain on self-diffusion in silver single crystals. At appreciable rates of strain the activation energy for diffusion fell to $\sim 0.7 \mathrm{eV}$., indicating this value as that required for the diffusion of vacancies produced by straining. Dr. M. C. Inman (National Physical Laboratory) gave some results for the effects of straining on the diffusion of antimony in copper and Dr. B. S. Berry (Fulmer Research Institute) for the energies for diffusion in quenched aluminium 4 per cent copper alloy.

Quenching of aluminium was discussed by M. M. Wintenberger (Vitry, France) both with reference to hardening and effects on electrical resistivity. Evidence was given which showed that annealing after a rapid quench gave a large residual resistivity and, because slight deformation reduced this, it was likely that it could be attributed to clustering of vacancies. The importance of clustering of vacancies in the 
hardening and subsequent softening of quenched sodium chloride crystals was emphasized by Dr. B. H. Kear (Tube Investments Research Laboratories). Describing work done at the University of Birmingham, he showed the effects of various quenching media, specimen sizes and quenching temperatures. After eliminating effects due to quenching strains there remained features closely analogous to those produced by single vacancies and vacancy clusters in metals.

Dr. T. H. Blewitt (Oak Ridge, temporarily at Harwell) referred to recent results in his own laboratory. Tensile tests on copper single crystals had been carried out at $20^{\circ} \mathrm{K}$. in a nuclear reactor after maintaining the specimens at that temperature throughout a bombardment of $4 \times 10^{17}$ neutrons. The hardening produced remained unchanged by pulse anneals up to about $250^{\circ} \mathrm{K}$. despite the large drop of extra resistivity at $\sim 35^{\circ} \mathrm{K}$. and its subsequent slow fall. Some slight decrease in critical shear stress occurred at $\sim 250^{\circ} \mathrm{K}$. but complete annealing did not occur until higher temperatures. New measurements of the energy associated with the $35^{\circ} \mathrm{K}$. resistivityannealing process yielded $0 \cdot 3 \mathrm{cal}$. / mole for a bombard ment of $4 \times 10^{17}$ neutrons. Dr. Blewitt suggested that these results, taken in conjunction with his own previously published work on the effects of alloying on the $35^{\circ} \mathbf{K}$. process in copper, precluded any simple interpretation of hardening as due to dispersed single vacancies and interstitial atoms. The annealing kinetics at $35^{\circ} \mathrm{K}$. were apparently first order with a range of activation energies, and might provisionally be ascribed to the migration of crowdions.

The mechanisms of irradiation-hardening of copper after neutron bombardment were discussed by Dr. M. J. Makin (Harwell). His experiments on single crystals and polycrystalline copper showed that there is a large and temperature-dependent increase in the stress required to generate dislocation loops; this must originate from the interaction of one or more of the products of bombardment (vacancies, interstitials, etc.) with dislocations. In addition, there is a general lattice hardening (independent of temperature) which makes it difficult to maintain the movement of dislocations; this could be attributed to the clustering of point defects or to the formation of sessile dislocations. Some preliminary results were given for the effects of electron irradiations on the strength of copper single crystals. Evidence for the dual nature of irradiation-hardening was also given by Dr. A. T. Churchman (A.E.I. Research Laboratories, Aldermaston) in an analysis of the dependence of neutron-bombardment hardening of mild steel upon grain size and temperature, and by Dr. A. D. Whapham (Atomic Energy Research Establishment, Harwell), who showed photomicrographs illustrating the propagation of slip in lithium fluoride crystals hardened by electron bombardment. Dr. R. E. Smallman (Attomic Energy Research Establishment, Harwell) gave results showing how $\mathrm{X}$-ray measurements indicate that a change occurs in the neutron bombardment of lithium fluoride at a critical dose of about $10^{17}$ nvt. Below this dose the lattice parameter increases with dose, but it remains constant above it; below, there are no diffraction streaks from single crystals and there is no line broadening and little low-angle scattering, but above $10^{17} \mathrm{nvt}$ all these increase. Again, this may indicate condensation of point defects at high doses.

Various considerations suggest that such alternating strain as occurs during fatigue tests may be a prolific source of vacancies. R. K. Ham (University of Birmingham) presented new results which showed that the fatigue hardening of copper single crystals at room temperature is somewhat analogous to that produced by irradiation. Plastic flow in a tensile test following a fatigue treatment starts in the fatigue strize, and then at a stress some 30 per cent higher than the fatigue stress (and typically about 50 times higher than the critical stress of the virgin crystal) there is a small yield drop and a Lüders band passes through the crystal. The temperature-dependence of this fatigue hardening is high. Less-detailed results obtained for zine crystals were described by Dr. G. B. Greenough (U.K. Atomic Energy Authority, Windscale). Dr. H. M. Rosenberg (University of Oxford) considered that electrical resistivity changes during fatigue of copper suggest that many more defects are created than during unidirectional deformation. The production of vacancies by unidirectional deformation is believed to be responsible for discontinuous flow in strain-ageing alloys : previous work has been done on dilute alloys, but S. G. Harris (University of Birmingham) showed how the Portevin-Le Chatelier effect in concentrated commercial and high-purity binary aluminium alloys is modified by a strain-induced precipitation process. This in turn can result in strikingly unusual 'shear' fractures after considerable". plastic extension.

Recent experiments on copper bombarded with $\alpha$-particles were described by Dr. R. S. Barmes (Atomic Energy Research Establishment, Harwell). On subsequent heating, bubbles of helium only appear where there is a prolific source of vacancies. The major sources of vacancies thus revealed are free surfaces and grain boundaries, with apparently only occasional dislocations. Dr. J. Nutting (University of Cambridge) suggested that, in technical creepresisting alloys, vacancies could be produced as a result of the volume changes involved in the dissolution of small precipitates or their change in composition.

The analysis of new results for the creep of copper between 400 and $750^{\circ}$ C. was discussed by Dr. P. Feltham (University of Leeds). Below a critical stress the rate of creep is controlled by the climb of edge dislocations, occasioned by the condensation of vacancies at jogs, and the activation energy is about $1.4 \mathrm{eV}$.; above the critical stress (identified as the strength of Cottrell-Lomer barriers) creep is controlled by the movement of screw dislocations containing jogs and the activation energy is about $2 \cdot 2 \mathrm{eV}$.

Dr. A. B. Lidiard (University of Reading) contributed a discussion of diffusion in ordered alloys, and Dr. R. W. Cahn (University of Birmingham) indicated how in such alloys creep phenomena depend markedly upon the degree of order near the critical temperature.

Some criticism of the theory of Williams and Hayfield was offered by Dr. T. P. Hoar (University of Cambridge), who thought that their derivation of logarithmic oxidation rates could depend on other rate-controlling processes. New evidence for the effects of point defects on physical properties was given by Dr. H. M. Rosenberg (heat conductivity in strained $\alpha$-brass single crystals) and by Dr. E. O. Hall (University of Sheffield) concerning effects of neutron-bombardment on the magnetic behaviour of single crystals of 2 per cent silicon ferrite: Fairly detailed analysis of the magnetic results was possible, and interpretations were offered in terms of an increase in density of dislocations and jogs and the creation of aggregates of point defects.
T. BroOM 